\title{
On Inverse Problem for Differential Operators with Deviating Argument
}

\author{
V. A. Yurko \\ Vjacheslav A. Yurko, https://orcid.org/0000-0002-4853-0102, Saratov State University, 83, Astrakhans- \\ kaya Str., Saratov, 410012, Russia, YurkoVA@info.sgu.ru \\ Second-order functional differential operators with a constant delay are considered. Properties of their \\ spectral characteristics are obtained, and a nonlinear inverse spectral problem is studied, which consists in \\ constructing operators from their spectra. We establish the uniqueness and develop a constructive procedure \\ for solution of the inverse problem.
}

Key words: differential operators, deviating argument, inverse spectral problem.

DOI: https://doi.org/10.18500/1816-9791-2018-18-3-328-333

\section{INTRODUCTION}

We study the inverse spectral problem for Sturm-Liouville differential operators with a constant delay. Such problems often appear in natural sciences and engineering (see, for example, monograph [1] and the references therein). Inverse spectral problems consist in constructing operators with given spectral characteristics. For the classical Sturm-Liouville operators the inverse problems have been studied fairly completely; the main results can be found in $[2,3]$. However, differential operators with delay are essentially more difficult for investigating, since the main methods in the inverse problem theory (the transformation operator method and the method of spectral mappings $[2,3]$ ) do not work for operators with delay. Note that some particular results on the inverse problems for operators with delay were obtained in [4-7].

Consider the boundary value problems $L_{j}(q), j=1,2$ :

$$
\begin{gathered}
-y^{\prime \prime}(x)+q(x) y(x-a)=\lambda y(x), \quad x \in(0, \pi), \\
y(0)=y^{\prime}(\pi)+H_{j} y(\pi)=0,
\end{gathered}
$$

where $\lambda$ is the spectral parameter, $a \in(0, \pi), q(x) \in L(a, \pi)$ is a complex-valued function, and $q(x) \equiv 0$ for $x \in[0, a]$. We study the inverse problem of constructing the potential $q(x)$ and the coefficients $H_{j}$ from the given two spectra of the boundary value problems $L_{j}(q)$. More precisely, let $\left\{\mu_{n j}\right\}_{n \geqslant 0}, j=1,2$ be the eigenvalues of the problems $L_{j}(q)$.

Inverse problem 1. Given $\left\{\mu_{n j}\right\}_{n \geqslant 0}, j=1,2$, construct $q(x)$ and $H_{j}$.

We note that in the case of large delay when $a \geqslant \pi / 2$, the characteristic functions of the problems $L_{j}(q)$ depend on the potential $q(x)$ linearly, i.e. the inverse problem becomes linear. This linear case was studied in [5,7]. For $a<\pi / 2$ the characteristic functions depend on the potential nonlinearly, i.e. the inverse problem becomes nonlinear. This nonlinear case is seriously more difficult for investigating and for constructing the global solution of the inverse problem. In this paper we study namely nonlinear case. For definiteness, let $a \in[2 \pi / 5, \pi / 2)$. The case $a<2 \pi / 5$ requires separate consideration. The main results of the paper are Theorem 1 and Algorithm 1, where a global constructive procedure for solving the inverse problem is provided, and the uniqueness of the solution is proved. 


\section{PROPERTIES OF SPECTRAL CHARACTERISTICS}

Let $S(x, \lambda)$ be the solution of Eq. (1) under the initial conditions $S(0, \lambda)=0$, $S^{\prime}(0, \lambda)=1$. Eigenvalues of the boundary value problem (1)-(2) coincide with the zeros of its characteristic function

$$
p_{j}(\lambda):=S^{\prime}(\pi, \lambda)+H_{j} S(\pi, \lambda), \quad j=1,2 .
$$

Lemma 1. Boundary value problem $L_{j}$ has a countable set of eigenvalues $\left\{\mu_{n j}\right\}_{n \geqslant 0}$, and for $n \rightarrow \infty$ :

$$
\sqrt{\mu_{n j}}=\left(n+\frac{1}{2}\right)+\frac{A_{0} \cos (n+1 / 2) a}{2 \pi n}+\frac{H_{j}}{\pi n}+o\left(\frac{1}{n}\right)
$$

where $A_{0}=\int_{a}^{\pi} q(t) d t$

Lemma 2. The specification of the spectrum $\left\{\mu_{n j}\right\}_{n \geqslant 0}$ uniquely determines $p_{j}(\lambda)$ via

$$
p_{j}(\lambda)=\prod_{n=0}^{\infty} \frac{\mu_{n j}-\lambda}{(n+1 / 2)^{2}}
$$

Let us study the connections between the characteristic functions $p_{j}(\lambda)$ and the potential $q(x)$. Let $\lambda=\rho^{2}$. The function $S(x, \lambda)$ satisfies the integral equation

$$
S(x, \lambda)=\frac{\sin \rho x}{\rho}+\int_{a}^{x} \frac{\sin \rho(x-t)}{\rho} q(t) S(t-a, \lambda) d t .
$$

Solving (6) we get for $x \geqslant 2 a$ :

$$
S(x, \lambda)=S_{0}(x, \lambda)+S_{1}(x, \lambda)+S_{2}(x, \lambda)
$$

where

$$
\begin{gathered}
S_{0}(x, \lambda)=\frac{\sin \rho x}{\rho}, \\
S_{1}(x, \lambda)=\int_{a}^{x} \frac{\sin \rho(x-t)}{\rho} q(t) S_{0}(t-a, \lambda) d t, \\
S_{2}(x, \lambda)=\int_{2 a}^{x} \frac{\sin \rho(x-t)}{\rho} q(t) S_{1}(t-a, \lambda) d t,
\end{gathered}
$$

Using (7) and (8), we calculate

$$
S_{1}(x, \lambda)=-\frac{\cos \rho(x-a)}{2 \rho^{2}} \int_{a}^{x} q(t) d t+\frac{1}{2 \rho^{2}} \int_{a}^{x} q(t) \cos \rho(x-2 t+a) d t .
$$

Denote $\Delta_{k}(\lambda):=S^{(k)}(\pi, \lambda), k=0,1$. In view of (3), one has

$$
p_{j}(\lambda):=\Delta_{1}(\lambda)+H_{j} \Delta_{0}(\lambda), \quad j=1,2,
$$


and consequently,

$$
\Delta_{0}(\lambda)=\frac{1}{H_{1}-H_{2}}\left(p_{1}(\lambda)-p_{2}(\lambda)\right) .
$$

Taking (7), (9) and (10) into account, we obtain

$$
\begin{aligned}
& \Delta_{0}(\lambda)=\frac{\sin \rho \pi}{\rho}-A_{0} \frac{\cos \rho(\pi-a)}{2 \rho^{2}}+\frac{1}{2 \rho^{2}} \int_{a}^{\pi} q(t) \cos \rho(2 t-\pi-a) d t+S_{2}(\pi, \lambda), \\
& \Delta_{1}(\lambda)=\cos \rho \pi+A_{0} \frac{\sin \rho(\pi-a)}{2 \rho}+\frac{1}{2 \rho} \int_{a}^{\pi} q(t) \sin \rho(2 t-\pi-a) d t+S_{2}^{\prime}(\pi, \lambda) .
\end{aligned}
$$

Denote

$$
\begin{aligned}
& \Delta_{0}^{*}(\rho):=2 \rho^{2}\left(\Delta_{0}(\lambda)-\frac{\sin \rho \pi}{\rho}+A_{0} \frac{\cos \rho(\pi-a)}{2 \rho^{2}}\right) \\
& \Delta_{1}^{*}(\rho):=2 \rho\left(\Delta_{1}(\lambda)-\cos \rho \pi-A_{0} \frac{\sin \rho(\pi-a)}{2 \rho}\right) .
\end{aligned}
$$

Then

$$
\begin{aligned}
& \Delta_{0}^{*}(\rho)=\int_{a}^{\pi} q(t) \cos \rho(2 t-\pi-a) d t+\delta_{0}(\rho), \\
& \Delta_{1}^{*}(\rho)=\int_{a}^{\pi} q(t) \sin \rho(2 t-\pi-a) d t+\delta_{1}(\rho),
\end{aligned}
$$

where $\delta_{0}(\rho)=2 \rho^{2} S_{2}(\pi, \lambda), \delta_{1}(\rho)=2 \rho S_{2}^{\prime}(\pi, \lambda)$. Using (9) and (10), we infer

$$
\begin{aligned}
& 2 \rho \delta_{0}(\rho)=-A \sin \rho(\pi-2 a)+\frac{1}{2} \int_{-(\pi-2 a)}^{(\pi-2 a)} Q(\xi) \sin \rho \xi d \xi \\
& 2 \rho \delta_{1}(\rho)=-A \cos \rho(\pi-2 a)-\frac{1}{2} \int_{-(\pi-2 a)}^{(\pi-2 a)} Q(\xi) \cos \rho \xi d \xi
\end{aligned}
$$

where

$$
\begin{gathered}
A=\int_{2 a}^{\pi} q(t) d t \int_{a}^{t-a} q(s) d s, \\
Q(\xi)=Q_{1}(\xi / 2+\pi / 2+a)-Q_{2}(\xi / 2+\pi / 2)-Q_{3}(\xi / 2+\pi / 2), \\
Q_{1}(x)=q(x) \int_{a}^{x-a} q(s) d s, \quad Q_{2}(x)=q(x) \int_{x+a}^{\pi} q(s) d s, \quad Q_{3}(x)=\int_{x+a}^{\pi} q(s) q(s-x) d s .
\end{gathered}
$$

For simplicity we assume that $q(x) \in A C[a, \pi]$. The general case requires small technical modifications. Denote $q_{1}(x):=q^{\prime}(x)$. Taking (15)-(18) into account, we get

$$
\begin{gathered}
4 \rho \Delta_{0}^{*}(\rho)=B_{1} \sin \rho(\pi-a)-2 A \sin \rho(\pi-2 a)- \\
-\int_{-(\pi-a)}^{(\pi-a)} q_{0}(\xi) \sin \rho \xi d \xi+\int_{-(\pi-2 a)}^{(\pi-2 a)} Q(\xi) \sin \rho \xi d \xi \\
4 \rho \Delta_{1}^{*}(\rho)=B_{2} \cos \rho(\pi-a)-2 A \cos \rho(\pi-2 a)+
\end{gathered}
$$




$$
+\int_{-(\pi-a)}^{(\pi-a)} q_{0}(\xi) \cos \rho \xi d \xi-\int_{-(\pi-2 a)}^{(\pi-2 a)} Q(\xi) \cos \rho \xi d \xi
$$

where $B_{1}=2(q(a)+q(\pi)), B_{2}=2(q(a)-q(\pi)), q_{0}(\xi)=q_{1}(\xi / 2+\pi / 2+a / 2)$. Denote

$$
\begin{aligned}
& d_{0}(\rho)=4 \rho \Delta_{0}^{*}(\rho)-B_{1} \sin \rho(\pi-a)+2 A \sin \rho(\pi-2 a), \\
& d_{1}(\rho)=4 \rho \Delta_{1}^{*}(\rho)-B_{2} \cos \rho(\pi-a)+2 A \cos \rho(\pi-2 a) .
\end{aligned}
$$

It follows from (19)-(20) and (21)-(22) that

$$
d_{0}(\rho)=-\int_{-(\pi-a)}^{(\pi-a)} R(\xi) \sin \rho \xi d \xi, \quad d_{1}(\rho)=\int_{-(\pi-a)}^{(\pi-a)} R(\xi) \cos \rho \xi d \xi
$$

where $R(\xi)=q_{0}(\xi)-Q(\xi)$, and $Q(\xi) \equiv 0$ outside the interval $(-(\pi-2 a),(\pi-2 a))$. In particular, this yields

$$
q_{1}(x)=R(2 x-\pi-a)+Q_{1}(x+a / 2)-Q_{2}(x-a / 2)-Q_{3}(x-a / 2), \quad x \in(3 a / 2, \pi-a / 2) .
$$

Denote by $\left\{\lambda_{n}\right\}_{n \geqslant 1}$ the zeros of the entire function $\Delta_{0}(\lambda)$. Then

$$
\sqrt{\lambda_{n}}=n+\frac{A_{0} \cos n a}{2 \pi n}+o\left(\frac{1}{n}\right) .
$$

\section{SOLUTION OF THE INVERSE PROBLEM}

In this section we present our main results: a constructive procedure for solving the inverse problem and the corresponding uniqueness theorem. The solution of Inverse problem 1 can be found by the following algorithm.

Algorithm 1. Let the spectra $\left\{\mu_{n j}\right\}_{n \geqslant 0}, j=1,2$ be given.

1. Construct $p_{j}(\lambda), j=0,1$, via (5).

2. Find $H_{1}-H_{2}$, using (4):

$$
H_{1}-H_{2}=\pi \lim _{n \rightarrow \infty}\left(\sqrt{\mu_{n 1}}-\sqrt{\mu_{n 2}}\right) .
$$

3. Construct $\Delta_{0}(\lambda)$, using (12), and calculate $\lambda_{n}$.

4. Find $A_{0}$ from (25).

5. Calculate $H_{1}$ and $H_{2}$, using (4).

6. Find $\Delta_{1}(\lambda)$ from (11).

7. Construct $\Delta_{j}^{*}(\lambda), j=0,1$, according to (13) and (14).

8. Calculate $A, B_{1}$ and $B_{2}$, using (19) and (12), and find

$$
q(a)=\left(B_{1}+B_{2}\right) / 4, \quad q(\pi)=\left(B_{1}-B_{2}\right) / 4 .
$$

9. Construct $d_{0}(\rho)$ and $d_{1}(\rho)$ by $(21)$ and $(22)$.

10. Calculate $R(\xi)$, using (23).

11. Find $q_{0}(\xi)$ for $\xi \in(-(\pi-a),-(\pi-2 a)) \cup(\pi-2 a, \pi-a): q_{0}(\xi)=R(\xi)$.

12. Calculate $q_{1}(x)=q_{0}(2 x-\pi-a)$ for $x \in(a, 3 a / 2) \cup(\pi-a / 2, \pi)$. 
13. Find

$q(x)=q(a)+\int_{a}^{x} q_{1}(t) d t, \quad x \in(a, 3 a / 2), \quad q(x)=q(\pi)-\int_{x}^{\pi} q_{1}(t) d t, \quad x \in(\pi-a / 2, \pi)$.

14. Using (24) and knowledge of $q(x)$ for $x \in(a, 3 a / 2) \cup(\pi-a / 2, \pi)$, construct $q_{1}(x)$ for $x \in(3 a / 2, \pi-a / 2)$ :

$$
\begin{aligned}
q_{1}(x)=R(2 x-\pi-a)+ & q(x+a / 2) \int_{a}^{x-a / 2} q(s) d s-q(x-a / 2) \int_{x+a / 2}^{\pi} q(s) d s- \\
& -\int_{x+a / 2}^{\pi} q(s) q(s-x+a / 2) d s .
\end{aligned}
$$

15. Calculate $q(x)$ for $x \in(3 a / 2, \pi-a / 2)$.

Thus, the following theorem is proved.

Theorem 1. The specification of two spectra $\left\{\mu_{n j}\right\}_{n \geqslant 0}, j=1,2$ uniquely determines the potential $q(x)$ and the coefficients $H_{1}, H_{2}$. The solution of Inverse problem 1 can be found by Algorithm 1.

Acknowledgements: This work was supported by the Ministry of Education and Science of the Russian Federation (project nos. 1.1660.2017/4.6) and by the Russian Foundation for Basic Research (projects nos. 16-01-00015, 17-51-53180).

\section{References}

1. Hale J. Theory of functional-differential equations. New York, Springer-Verlag, 1977. $420 \mathrm{p}$.

2. Freiling G., Yurko V. Inverse Sturm-Liouville Problems and Their Applications. New York, NOVA Science Publishers, 2001. 305 p.

3. Yurko V. Method of Spectral Mappings in the Inverse Problem Theory. Inverse and Illposed Problems Series. Utrecht, VSP, 2002. 316 p.

4. Freiling G., Yurko V. Inverse problems for Sturm-Liouville differential operators with a constant delay. Appl. Math. Lett., 2012, vol. 25, iss. 11, pp. 1999-2004. DOI: https://doi.org/10.1016/j.aml.2012.03.026

5. Vladičić V., Pikula M. An inverse problem for Sturm-Liouville-type differential equation with a constant delay. Sarajevo J. Math., 2016, vol. 12(24), no. 1, pp. 83-88. DOI: https://doi.org/10.5644/SJM.12.1.06

6. Yurko V., Buterin S., Pikula M. Sturm-Liouville differential operators with deviating argument. Tamkang J. Math., 2017, vol. 48, no. 1, pp. 61-71. DOI: https://doi.org/10.5556/j.tkjm.48.2017.2264

7. Buterin S., Yurko V. An inverse spectral problem for Sturm-Liouville operators with a large constant delay. Anal. Math. Phys., 2017, pp. 1-11. DOI: https://doi.org/10.1007/s13324-017-0176-6

\section{Cite this article as:}

Yurko V. A. On Inverse Problem for Differential Operators with Deviating Argument. Izv. Saratov Univ. (N.S.), Ser. Math. Mech. Inform., 2018, vol. 18, iss. 3, pp. 328-333. DOI: https://doi.org/10.18500/1816-9791-2018-18-3-328-333 


\title{
ОБ ОБРАТНОЙ ЗАДАЧЕ ДЛЯ ДИФФЕРЕНЦИАЛЬНЫХ ОПЕРАТОРОВ С ОТКЛОНЯЮЮЩМСЯ АРГУМЕНТОМ
}

\begin{abstract}
В. А. Юрко
Юрко Вячеслав Анатольевич, доктор фризико-математических наук, заведующий кафредрой математической фризики и вычислительной математики, Саратовский национальный исследовательский государственный университет имени Н. Г. Чернышевского, Россия, 410012, Саратов, Астраханская, 83, YurkoVA@info.sgu.ru

Рассматриваются фрункционально-дифрференциальные операторы второго порядка с постоянным запаздыванием. Установлены свойства их спектральных характеристик и исследуется нелинейная обратная спектральная задача, которая состоит в построении операторов по их спектрам. Доказана единственность решения обратной задачи и указана конструктивная процедура ее решения.

Ключевые слова: дисрференциальные операторы, отклоняющийся аргумент, обратная спектральная задача.
\end{abstract}

Благодарности. Работа выполнена при финансовой поддержке Минобрнауки РФ (проект № 1.1660.2017/4.6) и РФФИ (проекты № 16-01-00015, № 17-51-53180).

\section{Образец для цитирования:}

Yurko V. A. On Inverse Problem for Differential Operators with Deviating Argument [Юpко В. А. Об обратной задаче для дифференциальных операторов с отклоняющимся аргументом] // Изв. Сарат. ун-та. Нов. сер. Сер. Математика. Механика. Информатика. 2018. Т. 18, вып. 3. C. 328-333. DOI: https://doi.org/10.18500/1816-9791-2018-18-3-328-333 